\title{
An Effective Indirect Trefftz Method for Solving Poisson Equation in 2D
}

\author{
YOU Caixia* and ZHANG Guangde \\ School of Automobile and Traffic Engineering, Wuhan University of Science \\ and Technology, Wuhan 430081, China.
}

Received 15 December 2014; Accepted 8 June 2016

\begin{abstract}
In the solution domain, the inhomogeneous part of Poisson equation is approximated with the 5-order polynomial using Galerkin method, and the particular solution of the polynomial can be determined easily. Then, the solution of the Poisson equation is approximated by superposition of the particular solution and the Tcomplete functions related to the Laplace equation. Unknown parameters are determined by Galerkin method, so that the approximate solution is to satisfy the boundary conditions. Comparison with analogous results of others numerical method, the two calculating examples of the paper indicate that the accuracy of the method is very high, which also has a very fast convergence rate.
\end{abstract}

AMS Subject Classifications: 35A25

Chinese Library Classifications: O175.25

Key Words: Poisson equation; indirect Trefftz method; Galerkin method; boundary problem; numerical method.

\section{Introduction}

The Poisson equation has universal applicability, ranging from gravity, material physics and engineering to biochemical studies [1-4]. The exact analytical solutions, although useful in analyzing the phenomena under consideration, are almost impossible to achieve once the field equations, the boundary conditions or the geometry of the boundary deviate from being simple. Therefore, studying the numerical method is very meaningful.

The three main numerical techniques are the Finite Difference Method (FDM), the Finite Element Method (FEM), and the Boundary Element Method (BEM). The Finite Difference Method, which has been extensively applied to problems of fluid dynamics as well as stress analysis solves a problem as a pointwise approximation of its governing

${ }^{*}$ Corresponding author. Email address: 28978556@qq. com (C. X. You), gd-zhang@wust.edu.cn (G. D. Zhang) 
equations. However, the method is difficult to apply to problems involving irregular or complex geometric, and the solving process is always too complicate. The Finite Element Method can be easily applied for solving problems with complex geometries and has been successfully used for solving problems in fluid mechanics, potential flow, rock and solid mechanics and acoustics. However, since the method only provides an approximation to the solution, it is often necessary to use a large number of very small elements to obtain accurate results. By employing a very fine mesh, the number of nodal points and hence the size of the stiffness matrix, can increase rapidly, occupying a large amount of the computer's available storage space. The size of the global stiffness matrix, and hence the problems associated with it, can be reduced by employing a Boundary Element Method which reduces the dimensionality of the problem by one. Drawbacks of this method arise from the non-uniqueness of locations of the chosen singularities. In the numerical treatment, the method may be highly ill-conditioned.

Trefftz method is the boundary-type solution procedures using T-complete functions satisfying the governing equation. It was firstly proposed by Trefftz in 1926 ([5]). When applying the Trefftz method to the analysis of the boundary value problem of the Poisson equation, there is great difficulty due to the inhomogeneous term of the governing equation. If the inhomogeneous term is simple function, e.g. the unknown function is not included in it, the particular solution for the term can be derived easily and the Tcomplete functions can be defined from the homogeneous and particular solutions of the problem. If not so, e.g. the inhomogeneous term includes the unknown function, the particular solutions cannot be derived directly. In order to overcome this problem, this paper employs the Galerkin analysis scheme for the analysis. First, an inhomogeneous term is approximated by the polynomial function in the Cartesian-coordinates using Trefftz-type weighted residual method to determine the particular solution related to the function. Then, the solution of the Poisson equation is approximated by superposition of the particular solution and the T-complete functions related to the Laplace equation. Unknown parameters are determined using Galerkin method by letting the residual to be zero so that the approximate solution is to satisfy the boundary conditions. This formulization is based on the so-called indirect method. Since, in this case, no discretization is needed as well as the boundary integrals are regular, the algorithm is simple. Finally, the present method is applied to two numerical examples in order to examine the numerical properties of the present method.

\section{Theoretical foundations of the method}

Considering a two-dimensional Poisson equation

$$
\left\{\begin{array}{l}
\nabla^{2} u=\frac{\partial^{2} u}{\partial x^{2}}+\frac{\partial^{2} u}{\partial y^{2}}=b(x, y, u), \quad(x, y) \in \Omega, \\
u=\bar{u}, \quad(x, y) \in \Gamma_{1} \\
\frac{\partial u}{\partial n}=\bar{q}, \quad(x, y) \in \Gamma_{2}
\end{array}\right.
$$


where $\Omega, \Gamma_{1}$ and $\Gamma_{2}$ mean the object domain under consideration and the potential $u$ and the flux $q$ specified boundaries, respectively. Besides, $n$ and (-) denote the unit normal vector on the boundary, respectively.

Trefftz method is formulated with the non-singular T-complete functions which are determined so as to satisfy the governing equation. The functions related to the twodimensional Laplace equation in the bounded domain are given as follows

$$
\mathbf{u}^{*}=\left\{u_{1}^{*}, \ldots, u_{2 \mu-1}^{*}, u_{2 \mu}^{*}, \ldots\right\}^{T}=\left\{1, \ldots, r^{\mu} \cos (\mu \theta), r^{\mu} \sin (\mu \theta), \ldots\right\}^{T},
$$

where $\mathrm{r}$ and $\theta$ denote the plane polar coordinates whose origin is taken arbitrarily. From the definition of the T-complete functions, it can be found that $\mathrm{r} \mu \sin (\mu \theta)$ and $\mathrm{r} \mu \cos (\mu \theta)$ are the even function and uneven function about $\theta$, respectively. If the object domain is symmetrical about the origin, which will lead some boundary integrals constantly equal to zero and the completeness of the T-complete functions will lose. Therefore, the origin of coordinates should be put somewhere avoiding symmetry.

The potential $\mathrm{u}$ is approximated by superposition of finite number of T-complete functions $\mathrm{uj}^{*}$, which are exact solutions of the homogeneous parts of the Poisson equation and particular solutions of the inhomogeneous Poisson equations are added

$$
u \approx \hat{u}=a_{1} u_{1}^{*}+a_{2} u_{2}^{*}+\cdots+a_{N} u_{N}^{*}+u_{T}=\mathbf{a}^{\mathrm{T}} \mathbf{u}^{*}+u_{T},
$$

where $a_{j}(j=1, \cdots, N), u_{T}$ denotes the unknown parameter and the particular solutions of the inhomogeneous part, respectively. By differentiating Eq. (2.3) in the normal direction on the boundary, we have the approximate expression of the flux

$$
q \approx \hat{q}=\frac{\partial \hat{u}}{\partial n}=a_{1} q_{1}^{*}+a_{2} q_{2}^{*}+\cdots+a_{N} q_{N}^{*}+\frac{\partial u_{T}}{\partial n}=\mathbf{a}^{\mathbf{T}} \mathbf{q}^{*}+\frac{\partial u_{T}}{\partial n} .
$$

Since Eqs. (2.3) and (2.4) do not satisfy the boundary conditions of Eq. (2.1), the residuals yield

$$
\begin{cases}R_{1}=\hat{u}-\bar{u}=\mathbf{a}^{\mathbf{T}} \mathbf{u}^{*}+u_{T}-\bar{u} \neq 0, & u \in \Gamma_{1}, \\ R_{2}=\hat{q}-\bar{q}=\mathbf{a}^{\mathbf{T}} \mathbf{q}^{*}+\frac{\partial u_{T}}{\partial n}-\bar{q} \neq 0, & u \in \Gamma_{2} .\end{cases}
$$

In this paper, the unknown parameter a is determined so that the residual $R_{1}$ and $R_{2}$ are minimized simultaneously by the Galerkin method.

\section{In case of $b(x, y, u)=f(x, y)$}

An inhomogeneous term $f(x, y)$ is approximated with the 5-order polynomial of the certain coordinates $(x, y)$

$$
f(x, y) \approx c_{1}+c_{2} x+c_{3} y+\cdots+c_{20} x y^{4}+c_{21} y^{5}=\mathbf{c}^{\mathbf{T}} \mathbf{r},
$$


where $\mathrm{c}$ and $\mathrm{r}$ denote unknown parameter vector and the vector of all terms of the polynomial, respectively

$$
\begin{aligned}
& \mathbf{c}^{\mathbf{T}}=\left\{c_{1}, c_{2}, \cdots, c_{21}\right\}, \\
& \mathbf{r}^{\mathbf{T}}=\left\{1, x, y, x_{2}, x y, y^{2}, x^{3}, x^{2} y, x y^{2}, y^{3}, x^{4}, x^{3} y, x^{2} y^{2},\right. \\
&\left.x y^{3}, y^{4}, x^{5}, x^{4} y, x^{3} y^{2}, x^{2} y^{3}, x y^{4}, y^{5}\right\} .
\end{aligned}
$$

In the object domain, the approximation of the inhomogeneous term will yield residual

$$
R_{\Omega}=f(x, y)-\mathbf{c}^{\mathrm{T}} \mathbf{r} \neq 0, \quad(x, y) \in \Omega .
$$

Using Galerkin method, we have the weighted residual equation

$$
\int_{\Omega} \tilde{r} R_{\Omega} \mathrm{d} \Omega=\int_{\Omega} \tilde{r}\left(f(x, y)-\mathbf{c}^{\mathrm{T}} \mathbf{r}\right) \mathrm{d} \Omega=0,
$$

where $\tilde{r}$ is weighted function and given as:

$$
\tilde{r}=r_{i}, \quad i=1,2 \cdots 21 .
$$

Then, we have

$$
\mathrm{Dc}=\mathbf{g}
$$

where

$$
\begin{cases}d_{i j}=\int_{\Omega} r_{i} r_{j} \mathrm{~d} \Omega, & i, j=1, \cdots 21, \\ g_{i}=\int_{\Omega} r_{i} f(x, y) \mathrm{d} \Omega, & i=1, \cdots 21 .\end{cases}
$$

Solving (3.7), we have the unknown parameter vectorc, then Poisson's equation can be transformed into:

$$
\nabla^{2} u=\mathbf{c}^{\mathrm{T}} \mathbf{r}
$$

Assuming that the particular solution of the inhomogeneous part $r_{i}$ is $u_{i}^{p}$, from Eq. (2.2), we have the approximate solution of this kind of Poisson equation

$$
\hat{u}=\mathbf{a}^{\mathbf{T}} \mathbf{u}^{*}+c_{1} u_{1}^{p}+c_{2} u_{2}^{p}+\cdots+c_{21} u_{21}^{p}=\mathbf{a}^{\mathbf{T}} \mathbf{u}^{*}+\mathbf{c}^{\mathbf{T}} \mathbf{u}^{\mathbf{p}},
$$

where

$$
\nabla^{2} u_{i}^{p}=r_{i} .
$$

Since $r_{i}$ is the term of the polynomials $u_{i}^{p}$ can be determined easily (Appendix 1).

Obviously, Eq. (3.10) do not satisfy the boundary conditions of Eq. (2.1), the residuals yield

$$
\begin{cases}R_{1}=\hat{u}-\bar{u}=\mathbf{a}^{\mathbf{T}} \mathbf{u}^{*}+\mathbf{c}^{\mathbf{T}} \mathbf{u}^{\mathbf{p}}-\bar{u} \neq 0, & u \in \Gamma_{1}, \\ R_{2}=\hat{q}-\bar{q}=\mathbf{a}^{\mathbf{T}} \mathbf{q}^{*}+\mathbf{c}^{\mathbf{T}} \frac{\partial \mathbf{u}^{\mathbf{p}}}{\partial n}-\bar{q} \neq 0, & u \in \Gamma_{2} .\end{cases}
$$


Using Galerkin method, we have the weighted residual equation

$$
\int_{\Gamma_{1}} \frac{\partial \tilde{u}}{\partial n} R_{1} \mathrm{~d} \Gamma_{1}+\int_{\Gamma_{2}} \tilde{u} R_{2} \mathrm{~d} \Gamma_{2}=0
$$

where $\tilde{u}$ is weighted function and given as:

$$
\tilde{u}=u_{s}^{*}, \quad s=1 \cdots N .
$$

Then, we have

$$
\mathbf{K a}=\mathbf{f},
$$

where

$$
\begin{array}{ll}
k_{i j}=\int_{\Gamma_{1}} \frac{\partial u_{i}^{*}}{\partial n} u_{j}^{*} \mathrm{~d} \Gamma_{1}+\int_{\Gamma_{2}} u_{i}^{*} \frac{\partial u_{j}^{*}}{\partial n} \mathrm{~d} \Gamma_{2,} & \mathrm{i}, \mathrm{j}=1, \cdots, \mathrm{N}, \\
f_{i}=\int_{\Gamma_{1}} \frac{\partial u_{i}^{*}}{\partial n}\left(\bar{u}-\mathbf{c}^{\mathbf{T}} \mathbf{u}^{\mathbf{p}}\right) \mathrm{d} \Gamma_{1}+\int_{\Gamma_{2}} u_{i}^{*}\left(\bar{q}-\mathbf{c}^{\mathbf{T}} \frac{\partial \mathbf{u}^{\mathbf{p}}}{\partial \mathrm{n}}\right) \mathrm{d} \Gamma_{2}, & \mathrm{i}=1, \cdots, \mathrm{N} .
\end{array}
$$

Solving eqn (3.15) for the unknown parameter a and back-substituting the results into eqn (2.2) yield the solution of Poisson equation.

\section{$4 \quad$ In case of $b(x, y, u)=f(x, y)+g(x, y) u$}

In the same way, using Eqs. (3.1)-(3.3) to approximate $b(x, y, u)$, we have

$$
\begin{aligned}
& b(x, y, u)=f(x, y)+g(x, y) u=\mathbf{c}^{\mathbf{T}} \mathbf{r}, \\
& \hat{u}=\mathbf{a}^{\mathbf{T}} \mathbf{u}^{*}+u_{T}=\mathbf{a}^{\mathbf{T}} \mathbf{u}^{*}+\mathbf{c}^{\mathbf{T}} \mathbf{u}^{\mathbf{p}} .
\end{aligned}
$$

In this case, the unknown parameter $\mathrm{c}$ is the function of the unknown potential $u$ and therefore, more equation is necessary for determining $\mathbf{c}$. ual

In the object domain, the approximation of the inhomogeneous term will yield resid-

$$
R_{\Omega}=b(x, y, u)-\mathbf{c}^{\mathbf{T}} \mathbf{r}=f(x, y)+g(x, y)\left(\mathbf{a}^{\mathbf{T}} \mathbf{u}^{*}+\mathbf{c}^{\mathbf{T}} \mathbf{u}^{\mathbf{p}}\right)-\mathbf{c}^{\mathbf{T}} \mathbf{r} \neq 0, \quad(x, y) \in \Omega .
$$

And in the boundary of the domain, the approximation of $u$ and $q$ will yield residual

$$
\begin{cases}R_{1}=\hat{u}-\bar{u}=\mathbf{a}^{\mathbf{T}} \mathbf{u}^{*}+\mathbf{c}^{\mathbf{T}} \mathbf{u}^{\mathbf{p}}-\bar{u} \neq 0, & u \in \Gamma_{1}, \\ R_{2}=\hat{q}-\bar{q}=\mathbf{a}^{\mathbf{T}} \mathbf{q}^{*}+\mathbf{c}^{\mathbf{T}} \frac{\partial \mathbf{u}^{\mathbf{p}}}{\partial n}-\bar{q} \neq 0, & u \in \Gamma_{2} .\end{cases}
$$

By taking $\tilde{r}$ as the weight function of the residual Eq. (4.3), we have the weighted residual equation

$$
\int_{\Omega} \tilde{r} R_{\Omega} \mathrm{d} \Omega=\int_{\Omega} \tilde{r}\left(f(x, y)+g(x, y)\left(\mathbf{a}^{\mathbf{T}} \mathbf{u}^{*}+\mathbf{c}^{\mathbf{T}} \mathbf{u}^{\mathbf{p}}\right)-\mathbf{c}^{\mathbf{T}} \mathbf{r}\right) \mathrm{d} \Omega=0 .
$$


By taking $\tilde{q}$ and $\tilde{u}$ as the weight functions of the residuals $R_{1}$ and $R_{2}$, respectively, we have weighted residual equation

$$
\int_{\Gamma_{1}} \frac{\partial \tilde{u}}{\partial n} R_{1} \mathrm{~d} \Gamma_{1}+\int_{\Gamma_{2}} \tilde{u} R_{2} \mathrm{~d} \Gamma_{2}=0
$$

Combining Eqs. (4.5) and (4.6) and rearranging it, we have

$$
\left[\begin{array}{ll}
\mathbf{A}_{r c} & \mathbf{B}_{r a} \\
\mathbf{C}_{u c} & \mathbf{D}_{u a}
\end{array}\right]\left[\begin{array}{l}
\mathbf{c} \\
\mathbf{a}
\end{array}\right]=\left[\begin{array}{l}
\mathbf{f}_{r} \\
\mathbf{f}_{u}
\end{array}\right]
$$

where the $(21 \times 21)$ matrix $\mathbf{A}_{r c}$, the $(21 \times N)$ matrix $\mathbf{B}_{r a}$, the $(N \times 21)$ matrix $\mathbf{C}_{u c}$, the $(N \times$ $N)$ matrix $\mathbf{D}_{u a}$, the $(21 \times 1)$ vector $\mathbf{f}_{r}$ and the $(21 \times 1)$ vector $\mathbf{f}_{u}$ are defined as:

$$
\begin{array}{ll}
a_{i j}=\int_{\Omega} r_{i} g(x, y)\left(u_{j}^{p}-r_{j}\right) \mathrm{d} \Omega, & i, j=1, \cdots, 21, \\
b_{i j}=\int_{\Omega} r_{i} g(x, y) u_{j}^{*} \mathrm{~d} \Omega, & i=1, \cdots, 21 ; j=1, \cdots N, \\
c_{i j}=\int_{\Gamma_{1}} \frac{\partial u_{i}^{*}}{\partial n} u_{j}^{p} \mathrm{~d} \Gamma_{1}+\int_{\Gamma_{2}} u_{i}^{*} \frac{\partial u_{j}^{p}}{\partial n} \mathrm{~d} \Gamma_{2,} & i=1, \cdots, N ; j=1, \cdots, 21, \\
d_{i j}=\int_{\Gamma_{1}} \frac{\partial u_{i}^{*}}{\partial n} u_{j}^{*} \mathrm{~d} \Gamma_{1}+\int_{\Gamma_{2}} u_{i}^{*} \frac{\partial u_{j}^{*}}{\partial n} \mathrm{~d} \Gamma_{2,} & i=1, \cdots, N ; j=1, \cdots, N, \\
f_{r i}=-\int_{\Omega} r_{i} f(x, y) \mathrm{d} \Omega, & i=1, \cdots 21, \\
f_{u j}=\int_{\Gamma_{1}} \frac{\partial u_{j}^{*}}{\partial n} \bar{u} \mathrm{~d} \Gamma_{1}+\int_{\Gamma_{2}} u_{j}^{*} \bar{q} \mathrm{~d} \Gamma_{2}, & j=1, \cdots N .
\end{array}
$$

\section{Numerical examples}

\subsection{Example 1}

As a first example, we shall consider Poisson's equation on the ellipse $\Omega=\left\{x^{2} / 4+y^{2} \leq 1\right\}$, $b(x, y, u)=f(x, y)=-2$ with the boundary conditions $\left.u\right|_{\Gamma}=0$. The exact solution of this problem is given as

$$
u^{e x}=-\frac{4}{5}\left(x^{2} / 4+y^{2}-1\right) .
$$

This problem was chosen so we could compare our results with previously published results in [6-8]. The numerical results are presented in Table 1 with $N=10$ and $\eta=\left|\hat{u}-u^{e x}\right|$ is used as an error index which shows the computational accuracy of the proposed Trefftz method in this paper. The convergence estimation is performed by 
Table 1: The numerical results of example 1.

\begin{tabular}{||l|l|l|l|l|l|l|l||}
\hline$x$ & $y$ & $\begin{array}{l}\text { Analytical } \\
\text { solution }\end{array}$ & $\begin{array}{l}\text { MFS } \\
\text { solution[5] }\end{array}$ & $\begin{array}{l}\text { DRM } \\
\text { solution[6] }\end{array}$ & $\begin{array}{l}\text { H-b } \\
\text { solution[7] }\end{array}$ & $\begin{array}{l}\text { Trefftz } \\
\text { solution }\end{array}$ & $\eta$ \\
\hline 1.5 & 0.00 & 0.350 & 0.349 & 0.349 & 0.348 & 0.350 & $2.54 \mathrm{e}-014$ \\
\hline 1.2 & 0.35 & 0.414 & 0.418 & 0.418 & 0.419 & 0.414 & $2.64 \mathrm{e}-014$ \\
\hline 0.6 & 0.45 & 0.566 & 0.565 & 0.573 & 0.574 & 0.566 & $8.50 \mathrm{e}-014$ \\
\hline 0.0 & 0.45 & 0.638 & 0.637 & 0.646 & 0.646 & 0.638 & $2.99 \mathrm{e}-014$ \\
\hline 0.9 & 0.00 & 0.638 & 0.637 & 0.643 & 0.644 & 0.638 & $2.30 \mathrm{e}-014$ \\
\hline 0.3 & 0.00 & 0.782 & 0.781 & 0.789 & 0.790 & 0.782 & $3.11 \mathrm{e}-014$ \\
\hline 0.0 & 0.00 & 0.800 & 0.799 & 0.807 & 0.808 & 0.800 & $6.44 \mathrm{e}-015$ \\
\hline
\end{tabular}

$$
C_{u}=\frac{1}{M_{c}} \sum_{m=1}^{M_{c}}\left|\hat{u}-u^{E x}\right|
$$

where $M_{c}$ points are placed uniformly on the whole domain under consideration for evaluating. For this analysis, we take $M_{c}=20$. The convergence history of $C_{u}$ is shown in Fig. 1.

The abscissa and the ordinate denote the number of Trefftz function and $C_{u}$, respectively. It shows that $C_{u}$ converges very fast. The order of accuracy can reach $10^{-12}$ when just four Trefftz functions are taken. One notice that the convergence history of $C_{u}$ fluctuate slightly when $N$ become too big, that's because the condition number of the matrix $\mathbf{k}$ in Eq. (3.15) increases, which influence the accuracy of the solution, but the poor condition doesn't prevent the numerical results from converging towards the exact solution. Besides, in practice, $N$ always needn't be a big value considering the fast convergence property of Trefftz method in this kind of problem.

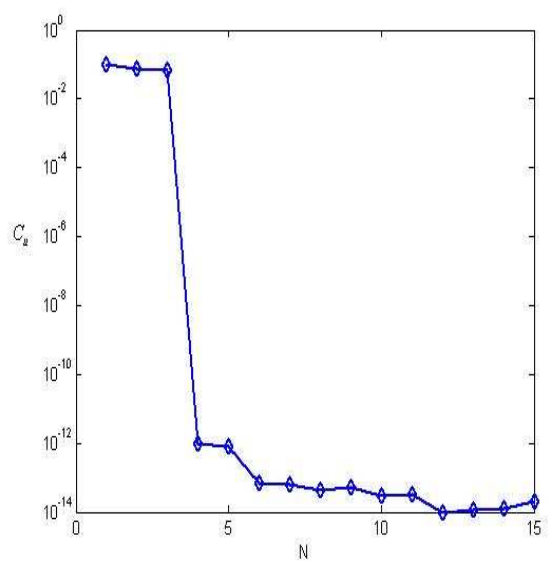

Fig. 1. Convergence property of $C_{u}$ 


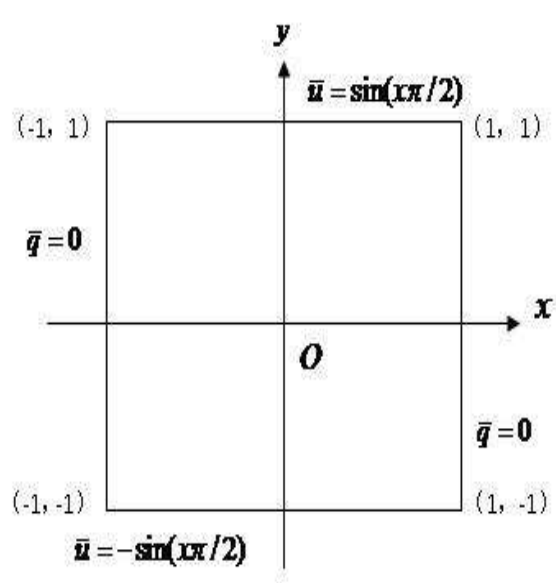

Fig. 2. Numerical example 2

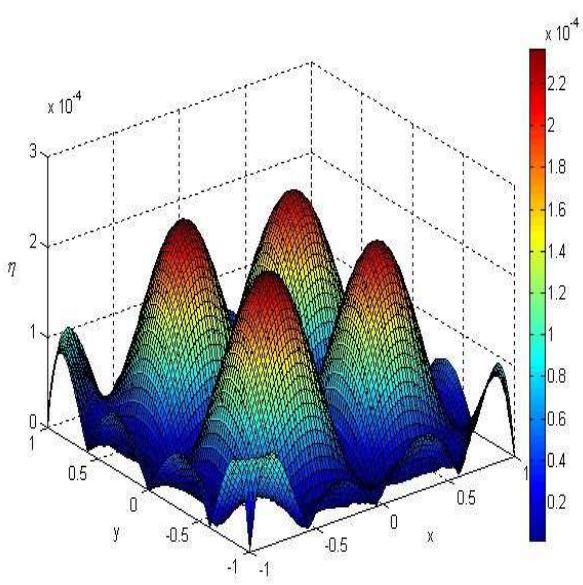

Fig. 3. The absolute error $\eta$ in the whole domain

\subsection{Example 2}

In the second example, as shown Fig. 2, we consider the problem on domain $\Omega=\{(x, y) \mid-$ $1 \leq x \leq 1,-1 \leq y \leq 1\}$ and $b(x, y, u)=f(x, y, u)=-\frac{\pi^{2}}{2} u$. The exact solution of this example is $u^{\text {ex }}=\sin \left(\frac{\pi}{2} x\right) \sin \left(\frac{\pi}{2} y\right)$.

Fig. 3 shows the absolute error $\eta$ in the whole domain in case of $N=15$. The convergence history of $C_{u}$ is shown in Fig. 4 From the convergence history, it shows that the order of accuracy can reach $10^{-4}$ when 11 Trefftz functions are taken, which is the same as that in [9] using 84 points.

$$
\bar{q}=0 x y \bar{u}=\sin (x \pi / 2) \bar{q}=0 \bar{u}=-\sin (x \pi / 2) O
$$

\section{Conclusion}

We have shown how to extend the indirect Trefftz method to solve Poisson equation without boundary or domain discretization. For overcoming the problem of inhomogeneous term, this paper presented the combination method of the Trefftz method and the Garlerkin analysis scheme. Two boundary value problems are considered as the numerical examples. A comparison with other methods indicates the high accuracy of this method. It's also illustrated through the examples that the method converges towards the exact solution.

\section{Acknowledgement}

The research is supported by National Natural Foundation of China (grant No. 50975212) at Wuhan University of Science and Technology. 


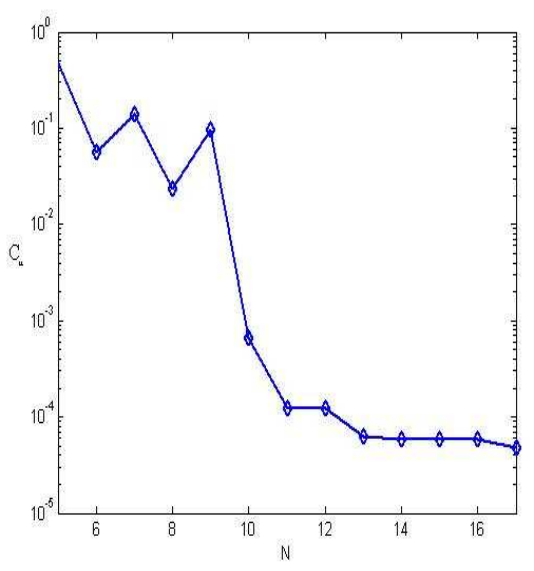

Fig. 4. Convergence property of $C_{u}$

\section{Appendix 1 Particular solution of Poisson equation $u_{i}^{p}$}

$u_{1}^{p}=\left(x^{2}+y^{2}\right) / 4 ;$

$u_{4}^{p}=\left(x^{4}+x^{2} y^{2}-y^{4} / 6\right) / 14 ;$

$u_{6}^{p}=\left(-x^{4} / 6+x^{2} y^{2}+y^{4}\right) / 14$;

$u_{8}^{p}=\left(x^{4} y+x^{2} y^{3}-y^{5} / 10\right) / 18$;

$u_{10}^{p}=\left(-x^{4} y / 2+x^{2} y^{3}+y^{5}\right) / 22$;

$u_{12}^{p}=\left(x^{5} y+x^{3} y^{3}-3 x y^{5} / 10\right) / 26$;

$u_{14}^{p}=\left(-3 x^{5} y / 10+x^{3} y^{3}+x y^{5}\right) / 26$;

$u_{16}^{p}=x\left(3 x^{6}+3 x^{4} y^{2}-5 x^{2} y^{4}+y^{6}\right) / 132$;

$u_{18}^{p}=\left(-x^{7} / 21+x^{5} y^{2}+x^{3} y^{4}-x y^{6} / 5\right) / 32$;

$u_{20}^{p}=\left(x^{7}-21 x^{5} y^{2}+35 x^{3} y^{4}+35 x y^{6}\right) / 1260$;

$$
\begin{aligned}
& u_{2}^{p}=\left(x^{3}+x y^{2}\right) / 8 ; \quad u_{3}^{p}=\left(x^{2} y+y^{3}\right) / 8 ; \\
& u_{5}^{p}=\left(x^{3} y+x y^{3}\right) / 12 ; \\
& u_{7}^{p}=\left(x^{5}+x^{3} y^{2}-x y^{4} / 2\right) / 22 ; \\
& u_{9}^{p}=\left(-x^{5} / 10+x^{3} y^{2}+x y^{4}\right) / 18 ; \\
& u_{11}^{p}=\left(x^{6}+x^{4} y^{2}-x^{2} y^{4}+y^{6} / 15\right) / 32 ; \\
& u_{13}^{p}=\left(-x^{6} / 15+x^{4} y^{2}+x^{2} y^{4}-y^{6} / 15\right) / 24 ; \\
& u_{15}^{p}=\left(x^{6} / 15-x^{4} y^{2}+x^{2} y^{4}+y^{6}\right) / 32 ; \\
& u_{17}^{p}=\left(35 x^{6} y+35 x^{4} y^{3}-21 x^{2} y^{5}+y^{7}\right) / 1260 \\
& u_{19}^{p}=\left(-x^{6} y / 5+x^{4} y^{3}+x^{2} y^{5}-y^{7} / 21\right) / 32 ; \\
& u_{21}^{p}=y\left(x^{6}-5 x^{4} y^{2}+3 x^{2} y^{4}+3 y^{6}\right) / 132
\end{aligned}
$$

\section{References}

[1] Hockney R. W., Eastwood J. W., Computer Simulation using Particles. Institute of Physics Publishing, 1988.

[2] Greenbaum A., Mayo A., Rapid parallel evaluation of integrals in potential theory on general three dimensional regions. J. Comput. Phys., 145 (1998), 731-745.

[3] Peskin C., Printz B., Improved volume conservation in the computation of flows with immersed elastic boundaries. J. Comput. Phys., 105 (1993), 33-45.

[4] LeVeque R. J., Li Z., The immersed interface method for elliptic equations with discontinuous coefficients and singular sources. SIAM J. Numer. Anal., 31 (1994), 1019-1031.

[5] Trefftz E., Ein Gegenstuck zum Ritzschen Verfahren. Proc 2nd Int Cong. Appl. Mech., Zurich, 1926: 131-147. 
[6] Golberg Michael A., The method of fundamental solution for Poisson's equation. Engineering Analysis with Boundary Elements, 16 (1995), 205-213.

[7] Partridge P. W., Brebbia C. A., The Dual Reciprocity Boundary Element Method. Computational mechanics publications, Southampton, 1992.

[8] Allesandri C. A., A spline based approach for avoiding domain integrations in the BEM. Comput. Struct., 41 (1991), 859-868.

[9] Kita Eisuke, Ikeda Youichi, Indirect Trefftz method for boundary value problem of Poisson equation. Engineering Analysis with Boundary Elements, 27 (2003), 825-833. 